\title{
gs \\ Exploring the context of change: Understanding the kinetics of a studio physics implementation effort
}

\author{
Patrick J. Enderle* \\ Center for Education Research in Mathematics, Engineering, and Science, College of Education, The Florida State University, \\ Tallahassee, Florida 32306, USA \\ Sherry A. Southerland \\ FSU-Teach, G119 STB, The Florida State University, Tallahassee, Florida 32306, USA \\ Jonathon A. Grooms \\ Center for Education Research in Mathematics, Engineering, and Science, College of Education, The Florida State University, \\ Tallahassee, Florida 32306, USA
}

(Received 5 October 2012; published 10 April 2013)

\begin{abstract}
The SCALE-UP studio physics class involves the physical redesign of a classroom to encourage more collaborative interactions and student-centered teaching, an approach shown to increase student learning on several different measures. However, research into the contextual issues involved in implementing a studio course using the SCALE-UP model remains limited. The research presented here explores the impact of situational factors on the implementation and maintenance of a research-based instructional innovation in a large research university. The specific focus of this investigation was the development and implementation of a studio version of an introductory physics course sequence at a large research university in the southeast United States using the SCALE-UP model. Interview, observation, and artifact data (including documents and Email conversations), collected over a period of two years, were analyzed. Using the Teacher Centered Systemic Reform framework, the data were analyzed to develop themes describing forces that both encouraged and restricted the growth of this studio course. Influential forces operated at the classroom, department, university, and broad cultural levels. The results demonstrate the importance of considering the specific nature of innovations implemented and their alignment with desired learning goals and outcomes. The importance of faculty collegiality emerges as a critical departmental force, as does administrative intervention at the department and university level. Broader, societal conversations related to improving undergraduate science education also provided important contextual framing for the change effort studied. The findings highlight important factors to contemplate when undertaking similar change efforts and recommendations from this study are offered for consideration.
\end{abstract}

DOI: 10.1103/PhysRevSTPER.9.010114

\section{INTRODUCTION}

Physics education research (PER) comprises several decades of well-established research exploring the conceptual difficulties students face in learning introductory physics at the postsecondary level and a variety of approaches that can be used to address those challenges [1]. These approaches range in size and scope to encompass specific activities, instructional strategies, defined curricula, as well as entire course redesigns. This vast assemblage provides faculty members with many options to explore to enhance their teaching of fundamental physics concepts.

\footnotetext{
*Corresponding author.

Published by the American Physical Society under the terms of the Creative Commons Attribution 3.0 License. Further distribution of this work must maintain attribution to the author(s) and the published article's title, journal citation, and DOI.
}

PACS numbers: 01.40.Fk, 01.40.Di, 01.40.gb, 01.40.jc

Dissemination of this information and guidance has been effective in reaching individual physics instructors [2]. However, adoption and implementation efforts have not developed in a concurrent manner [2-4]. Although a great deal of research explores individual faculty members making personal changes to their own teaching to improve student learning, research into broader institutional efforts focused on understanding educational innovations involving multiple instructors and combinations of resources remains limited [5].

Much of the research on instructors' efforts in implementing and maintaining PER innovations has focused on personal factors that influence a faculty member's adoption of instructional strategies [6]. Research in undergraduate science education has demonstrated the importance of instructors' beliefs about science teaching and learning in attempting changes in their pedagogical practices [7-9]. An instructor's use of these practices has been shown to be a reciprocally important factor in the further development 
of their beliefs; indeed, instructors' beliefs and practices involved in the change in teaching are recursively related $[2,10]$. Although the instructor is critical to the interpretation and enactment of innovations in teaching, such efforts do not occur in a vacuum. Rather, efforts at changing physics teaching are embedded in complex institutional structures, with multiple influential forces coming to bear on them. An understanding of the role of these forces on adoption efforts remains underrepresented in the research literature, with several authors calling for more research into the contextual influences on implementing innovations and their compatibility within these systems $[2,3,6,9,11]$.

Research on situational factors influencing implementation of research-based instructional strategies must employ theoretical perspectives that accommodate the multiple layers of organization present in postsecondary education. Current reviews of such work emphasize the use of a systems framework for making sense of these overlapping groupings, each imbued with their unique cultural expectations and inputs for change efforts [3]. A systems framework accounts for multiple levels of institutional organization, including departmental, college, university, and societal level considerations. However, researchers have demonstrated a concurrent need to consider the nature of instructors' beliefs about teaching and practices in their classrooms within these systems [8,9]. The use of theoretical frameworks bridging different education research groups have shown promise in making sense of efforts to change education at the undergraduate level [9]. The Teacher Centered Systemic Reform (TCSR) framework offers a useful theoretical approach to studying efforts in undergraduate innovation by incorporating classroom interactions and teacher beliefs into the analysis of several contextual spheres of influence on undergraduate instruction $[8,9]$.

This study explores the impact of situational factors on the implementation and maintenance of a research-based instructional innovation in a large research university. The physics department at this university implemented and maintains a studio physics course program. The program includes several sections of their introductory, calculusbased course sequence (two sections of a force and motion course, one section of the electricity and magnetism course) that are taught using the SCALE-UP model [12] alongside the more traditional lecture-laboratory-recitation course combination. The SCALE-UP model incorporates a physical redesign of the classroom space with a combined teaching approach that addresses content through a varied mixture of student group work on conceptual problems and physics investigations interspersed with short lectures on relevant topics.

A team of four faculty members, Albert, Isaac, Max, and Robert (all pseudonyms), share the responsibility for these courses, three of whom are responsible for the creation and implementation of the studio course program. The study presented here employs a systematic theoretical framework to analyze observation, interview, and document data collected from this group of faculty over the course of two years. The analysis determined situational factors that were perceived by the studio faculty group and observed by the researcher to be the most crucial in supporting their efforts at maintaining a studio physics course program. The results also highlight contextual factors that limited the instructors' efforts or served as barriers to continuation. Based upon these findings and other works $[3,5]$, recommendations are discussed concerning institutional actions that can be used as levers to alleviate some of the resistive factors and enhance the supportive ones.

\section{THEORETICAL BACKGROUND}

\section{A. Studio physics courses}

Studio physics courses have a well-established history in the PER community [1], emerging from research contexts at the University of Washington Physics Education Research Group and published curricula such as Workshop Physics [13]. Studio physics courses represent innovation over traditional, lecture-based courses by engaging students in activities focused on developing conceptual understanding rather than transmission of canonical physics knowledge. These courses have been adapted to other scientific disciplines as well. One of the most important innovations in studio courses involves the redesign of the classroom space that facilitates a change in the nature of instruction. Yet, the creation of the space alone does not automatically result in a more effective learning environment.

Further work exploring the efficacy of the Rensselaer studio model demonstrated the impact of curricular choices on student learning in studio courses [14]. The use of research-based curricula that have demonstrated enhanced learning gains lead to significantly greater learning gains in studio physics courses than in studio courses that did not employ such materials. These findings demonstrate that implementation of a studio course model involves multiple, subtle considerations to engender the desired increase in student learning. Yet, as studio programs continue to develop at institutions around the country, class size has become a factor in making this course format affordable and efficient for larger institutions.

The SCALE-UP program at North Carolina State University, emerging from the physics education research group, has developed a studio model capable of serving classes with over 100 students [12]. Over 50 institutions, including several large research universities like MIT, have adopted the SCALE-UP model for teaching an array of STEM courses. The lecture and laboratory sections of a physics course are combined in the SCALE-UP model, with students meeting several times a week in extended class sessions, totaling about five to six hours in a week. The SCALE-UP model necessitates a unique physical 
space that has anywhere from six to fifteen circular worktables in a room, each equipped with several networked computers. Collaborative groups of students are assembled, several at each worktable. Students engage in several types of activities through group collaboration, with the responsibility for completing assignments and developing physics understanding placed on the students, while the teacher and assistants act as additional resources and guides [12]. These activities include conducting data collecting investigations, interactive simulations, and solving complex applied problems. Class activities are punctuated with discussions and brief lectures that highlight and clarify fundamental concepts. The goal for the SCALE-UP course model is to create an environment where students are actively engaged in learning content through interactions with students, faculty, and collaborative work, rather than passive recipients of information and direction.

The adoption of the SCALE-UP model at different institutions has produced a body of evidence demonstrating its success in enhancing physics teaching and student learning. In a study looking across data from multiple institutions, the normalized learning gains for students in studio physics courses were found to be significantly higher than students who received lecture-based instruction in a variety of contexts [12]. Failure rates in both introductory physics courses and advanced courses that require introductory physics course work were significantly lower for SCALE-UP students than for lecture students. Courses implementing the SCALE-UP model also demonstrated improvements in attendance, attitude towards science, and improvement in females' achievement in physics [11]. However, further study is warranted if the physics education community is to understand how contextual factors influence the adoption and implementation of studio courses using the SCALE-UP model $[1,11]$.

\section{B. Change in undergraduate contexts}

To understand contextual factors that influence the adoption and implementation of research-based educational innovations, such as studio physics courses, research must incorporate approaches that acknowledge and explore the multifaceted context in which these efforts occur. Large research universities are complex systems with the work of several institutional entities connected to any single course. Thus, change in a particular course necessitates consideration of resonant effects on the institutional entities supporting it.

Reviews of the knowledge base regarding improving undergraduate science education have noted prevalent barriers to and potential levers for realizing educational changes within these systems $[3,9,11]$. That said, these reviews also call for additional work in understanding specific cases of successful change efforts to elucidate which barriers were most prevalent and how resources were leveraged to overcome them. The systems framework argued for in one review [3] serves as a useful guide to begin exploring the influential structural levels of large research universities. Nevertheless, this approach omits some considerations that others have demonstrated as critical components to any change effort, which include the classroom level and teachers' knowledge and beliefs [9].

\section{The Teacher Centered Systemic Reform framework}

The TCSR framework [8], a useful theoretical perspective to consider change efforts within a complex educational system, positions the instructor, with his or her history, beliefs, and practices, as the central component of the effort that reciprocally influences the other levels. The TCSR model was developed from studies of undergraduate reform efforts, and accounts for the intertwined

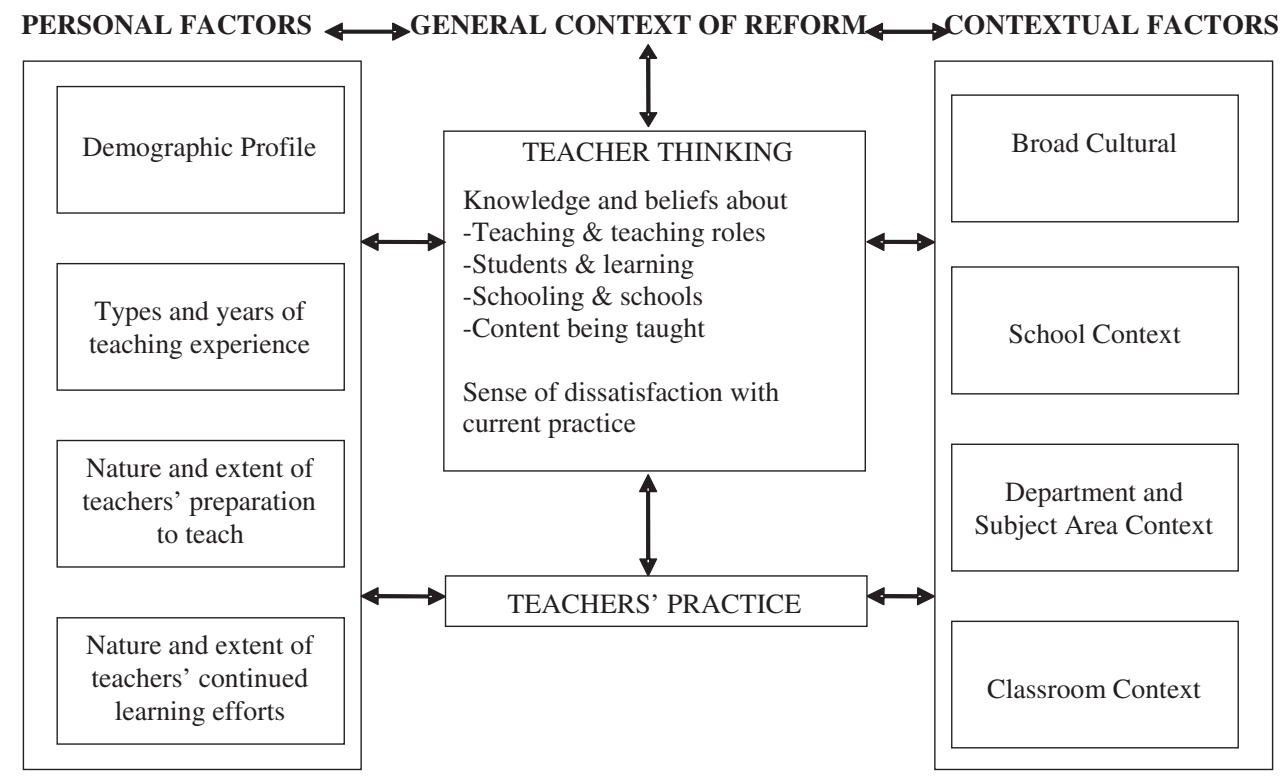

FIG. 1. The Teacher Centered Systemic Reform (TCSR) framework [8]. 
system of organization that is common in large research universities. The centrality of the instructor in the model offers a necessary perspective as the teacher is the ultimate arbiter of any such implementation effort, thus their perception of and response to contextual influences highlights those of importance. The model also incorporates other personal factors that shape the central role of an instructor, including professional development and years of experience in teaching (Fig. 1).

The utility of the TCSR framework emerges from the incorporation of several contextual levels in analytical consideration of the influences experienced by teachers involved in change efforts. Classroom level considerations can involve issues arising from the physical organization of a classroom space, curricular materials used, and the types of students and interpersonal interactions that occur.

The department level of contextual analysis could encompass the type of administrative support offered, the culture among colleagues, and course scheduling logistics. Moving analysis to the university level, administrative actions, institutional status, and relationships among component programs can serve as key focal points for understanding the trajectory of change efforts. Finally, the broader cultural context can be examined for the impact of professional associations, societal goals for postsecondary education, and the prevalence of acknowledged resources.

\section{METHODS}

\section{A. Studio physics faculty group}

This study occurred at a large, public research university in the southeast United States. The location and nature of the institution were defining factors in this study. During the time of the study, the state in which the university resided experienced dramatic budget shortages, leading to several years of reduction in budget allocations. This reality created a difficult financial environment for university operations, with many established programs being shuttered, downsized, or merged. The high research activity taking place at this institution also created a specific context for this study, as research activity provided significant financial support for some programs and created a particular culture of expectations among administrators and faculty members [15]. The Department of Physics at this university maintained a significant amount of funding from federal agencies and other sources, particularly through partnerships with several large internationally recognized laboratories.

To understand the role of contextual influences on the development of the studio physics courses, the four faculty members who worked together on those courses served as the research subjects for this study. Albert, Isaac, and Robert comprised the original faculty group that created the studio physics program. All three were long-term, tenured physics department faculty members with established research programs. They also served in a variety of administrative capacities, at both the department and university level. Albert served as the chair of the department during the initial phases of development, yet had stepped down from that position by the time this study commenced. Robert previously served as director for the university honors program, and he was serving as the director of undergraduate studies for the department at the time of this study. Isaac had served as the course director for several of the large introductory courses in the department. During the time of this study, the fourth member of the studio physics faculty group, Max, began his work with the group soon after being granted tenure.

\section{B. Data collection and analysis}

The time frame for this study extended through 2009 to 2011 and during this time data were collected from a wide range of sources, including interviews, observations, and document analysis. Interviews were conducted with the four group members, both individually and as a group. All interviews employed a semistructured question protocol, providing opportunities for follow-up exploration of ideas, resulting in a total of over seven hours of interview data. The individual interviews familiarized the researchers with the faculty members and in some cases provided historical information. The group interviews provided more information about how the group collaborated and their shared perspective in regards to influences on the studio physics program.

Observations of various interactions, both inside and outside the classroom, allowed for insight into the practices of the group members and their responses to external influences. These observations also afforded the researchers insight into how certain external stimuli shaped the nature of the studio course. Overall, 105 separate events were observed and recorded. Another data source for the study involved document analysis, the bulk of which was composed of a collection of 44 shared Email conversations involving the four instructors. These Emails included both internal discussions among the studio physics group and their communications with other individuals regarding the studio physics program. All interviews and observation notes were digitally transcribed for analysis purposes. The data analysis phase of this study employed a systematic coding procedure known as the constant comparison method [16]. This analysis began with the establishment of a preliminary coding schema with several broad codes developed in relation to the TCSR framework [8]. However, the analysis remained open to allow for the emergence of new codes and categories that may not be represented in the initial coding scheme. All three types of data were used during the analysis phase.

The coding analysis process involved three primary stages of activity, as defined by Strauss and Corbin [16]: open coding, axial coding, and selective coding. During the 
TABLE I. Example codes used during the three phases on constant comparison data analysis (I-initial coding scheme, E- emerged during analysis, D-discarded during analysis).

\begin{tabular}{|c|c|c|c|}
\hline Contextual level & Open coding & Axial codes & Selective theme \\
\hline Classroom & $\begin{array}{l}\text { Learn-by-doing (E) } \\
\text { Conceptual understanding (I) } \\
\text { Room geometry (I) } \\
\text { Group work (E) } \\
\text { Technology-online problems (I) } \\
\text { Clickers (D) }\end{array}$ & SCALE-UP elements & $\begin{array}{l}\text { Alignment of goal and inno- } \\
\text { vation (motive) }\end{array}$ \\
\hline Department & $\begin{array}{l}\text { Chair-support (I) } \\
\text { Chair-resistance (D) } \\
\text { FCI evidence (E) } \\
\text { Funding help (E) } \\
\text { Pride in traditional teaching (D) } \\
\text { Other change efforts (I) } \\
\text { FCI evidence (E) } \\
\text { Colleague ambivalence (E) }\end{array}$ & Administrative support & $\begin{array}{l}\text { Supportive culture allowing } \\
\text { innovation (motive) }\end{array}$ \\
\hline University & $\begin{array}{l}\text { Direct funding infusion (E) } \\
\text { Grant competition (E) } \\
\text { Other innovative programs (I) } \\
\text { Budget issues (I) } \\
\text { Registrar (I) } \\
\text { Room allotment (E) } \\
\text { Research pressure (D) } \\
\text { Other academic units (I) }\end{array}$ & Limited funding sources & $\begin{array}{l}\text { Resource allocation and com- } \\
\text { petition (restrictive) }\end{array}$ \\
\hline Cultural & $\begin{array}{l}\text { Education research (E) } \\
\text { Professional associations (I) } \\
\text { Curriculum options (I) } \\
\text { Teacher education (E) } \\
\text { PR or press attention (I) } \\
\text { Peer institution comparisons (I) } \\
\text { North vs South (D) }\end{array}$ & $\begin{array}{l}\text { Wealth of PER based } \\
\text { resources }\end{array}$ & $\begin{array}{l}\text { Need for implementing } \\
\text { STEM education innovations } \\
\text { (motive) }\end{array}$ \\
\hline
\end{tabular}

initial coding stage, codes generated from the TCSR framework and those that emerged from the review of data transcripts were assigned to discreet pieces of information within those transcripts. The next stage, known as axial coding, involved assigning and condensing the original assemblage of codes into progressively larger codes, applying those codes to the various data sources. The final stage, selective coding, involved making further connections with the axial code structures, ultimately resulting in broader thematic findings. At all three stages, any codes that were no longer applicable or discounted by other evidence were discarded.

The results generated from this analysis were further validated through a review process involving the four studio physics faculty members. The researchers met with Albert, Isaac, Max, and Robert collectively to review the findings and discussion. The faculty members offered suggestions for minor revisions, mostly grammatical, but supported the analysis and results developed by the researchers. Table I provides examples of codes that were generated initially and during analysis. This table is not comprehensive but is intended to provide the reader insight into the stages of data analysis.

\section{RESULTS}

The systematic organization of contextual forces in the TCSR framework provided an approach for identifying important influences over varied time frames. In studying complex systems, such as a change effort in a multilayered research university, the salient forces can change over time, with some levels being more significant at certain stages and less influential during others. In order to capture these dynamics, the analysis was separated into several periods of activity representing important stages where the interactions of different contextual forces shifted to produce different effects upon the studio physics program.

Figure 2 offers a diagram to represent the developmental trajectory of the studio physics program over time. The studio program can be considered the "object in motion" in this chart. The program experienced different periods of development and expansion, which are reflected in the 


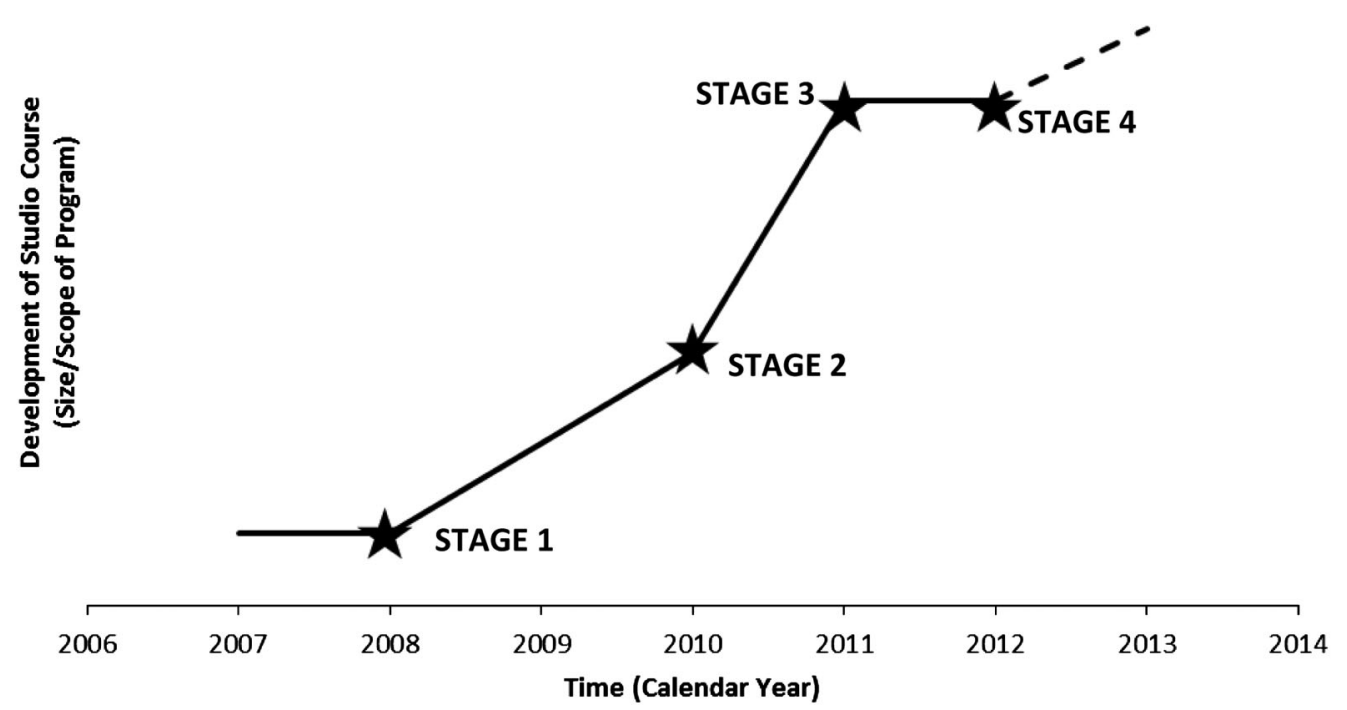

FIG. 2. Kinetics of the studio course effort: The developmental trajectory of the studio physics program is represented by this line graph plotting the change in size of the program over the course of several years.

periods of acceleration. For several critical stages of activity (stages 1-4), a free body diagram has been developed to describe the nature of the forces acting on the object at that point in time. The different forces identified are also aligned with the contextual levels described in the TCSR framework. For each level in each stage, a "motive" force $\left(F_{M}\right)$ represents actions that helped further the studio physics program, and a "restrictive" force $\left(F_{R}\right)$ describes activities that limited the development of the studio program. (For example, Fig. 3 is the free body diagram for stage 1.) Each stage diagram has an accompanying description of the critical events and influences operating on the studio physics program at that time.

\section{A. Stage 1: Development}

The development of the studio physics course effort was initiated through Robert's interest in various PER efforts around the United States. This interest led to him talking with Albert and Isaac about the potential of some of these innovations, particularly the SCALE-UP program. The forces that interacted to bring the studio effort to this stage acted across multiple contextual levels. That is, issues that arose at the classroom level shaped events that occurred at other levels, such as the department level.

The restrictive forces important in stage 1 stem from the physical layout of the classrooms and lecture halls in which introductory physics courses were taught (see Fig. 3). In these classroom settings, the faculty members were not able to engage with their students on a more individual basis, and students demonstrated typical behaviors they are trained to enact in such spaces (i.e., passive listening to a person in front that leads to distractions from a variety of sources). This sense of disengagement and the concomitant lack of physics learning fostered a sense of pedagogical discontentment, the dissatisfaction that the faculty experienced when the results of their instruction failed to meet their goals [17]. This discontentment provided motive force for the three tenured, veteran members of the department to come together as a collaborative team to improve introductory physics instruction. The support and combined energy of the veteran group became a primary motive force for this group at the departmental level.

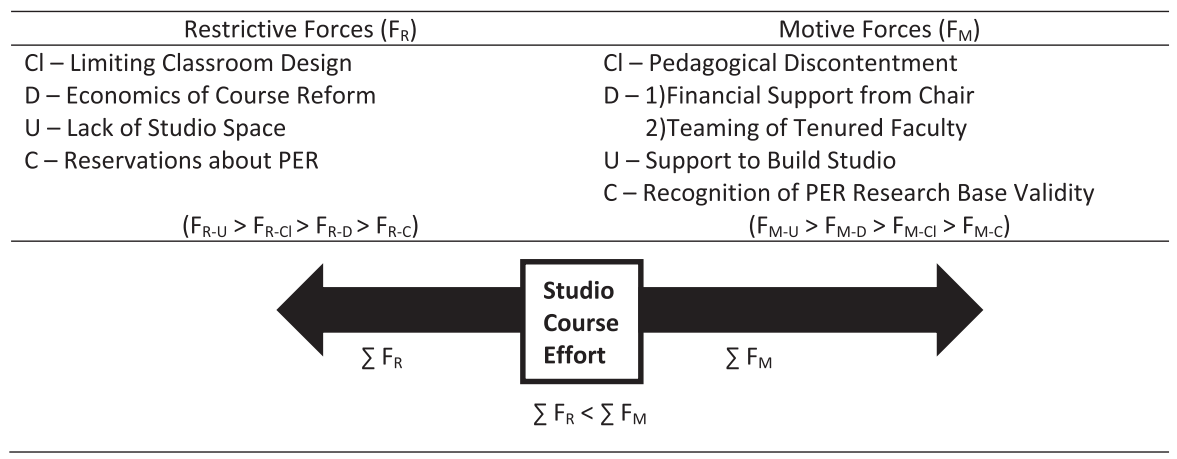

FIG. 3. Free body diagram for stage 1: Development of the studio physics program ( $\mathrm{Cl} —$ classroom; D—department; U—university; C-culture). 
This group is made up of people that are forward thinking about teaching techniques and also deeply concerned about whether the students in their class actually learn something. (Isaac, group interview)

Although the team of faculty worked together to move the studio course effort forward, they faced perhaps the largest resistance from the lack of an actual studio space existing on the campus that met the specifications described in the SCALE-UP model. However, through discussions with colleagues from several departments across campus, a campaign supporting the inclusion of one studio space in a new classroom building successfully garnered university administrators' approval. The support for and establishment of the required classroom space for the studio course served as the greatest motive force for this group at the university level.

As the forward motion of the studio course effort continued with the building of the space, another resistive force emerged at the departmental level. The changes required to realize a studio version of an introductory physics course demanded a notable investment of resources, in terms of both physical equipment and faculty scheduling. In response, Albert, Isaac, and Robert were able to design a unique course schedule and size that produced a similar faculty time to student served ratio as the traditional lecture-laboratory-recitation combination already present in the department. "The studio [course] is cheaper than the traditional lecture class in this department." (Robert, group interview) The economic feasibility of the studio model provided convincing arguments for department colleagues who may have been reticent to support the studio effort. The financial resistance of equipment costs was alleviated by another motive force that came in the form of a one-time investment of department funds by the department chair, combined with the award of a small university level course improvement grant.

So we got the grant and Albert, who was chairman at the time, came into my office one day. He said "Isaac, if you had to spend [several thousand dollars] in the next 24 hours, could you do it?" I said, "I've got the item numbers!" (Isaac, group interview)

With university and departmental administrative support, the faculty team was able to develop and establish an economically feasible studio model in a newly built studio classroom. This developmental stage was situated in a broader, cultural interaction in which physicists were questioning the need for PER innovations in light of competing time and resource demands contrasted by an acknowledgement of the validity of the PER research findings (an acknowledgement fostered by the fact that physicists were the ones conducting the physics education research). Thus, in the spring of 2008, the first introductory studio physics course focusing on forces and motion concepts commenced. The studio course continued to grow over the next year, with increasing student enrollment and the team developing a studio version of the second introductory course focusing on electricity and magnetism. These courses remained calculus based like the more traditional sections of lecture and laboratory courses, and they served similar student populations, primarily science and engineering majors.

\section{B. Stage 2: Expansion}

Once the two studio courses were established, the developmental trajectory continued to moderately accelerate with both being offered in the fall and spring semesters. The first introductory course, which was a required physics course for many degree programs, quickly gained in popularity, attracting students interested in the learning format and others who perceived the approach to be less difficult. This variation in the student populations of the courses in terms of abilities and aptitudes emerged as a resistive force for the faculty group's efforts. Yet, Albert, Isaac, and Robert dealt with the challenge of students' disparate abilities through a curricular flexibility inherent in the SCALE-UP model. (See Fig. 4 for the free body diagram for stage 2.)

The SCALE-UP model focuses on the physical design of the classroom space and the general nature of

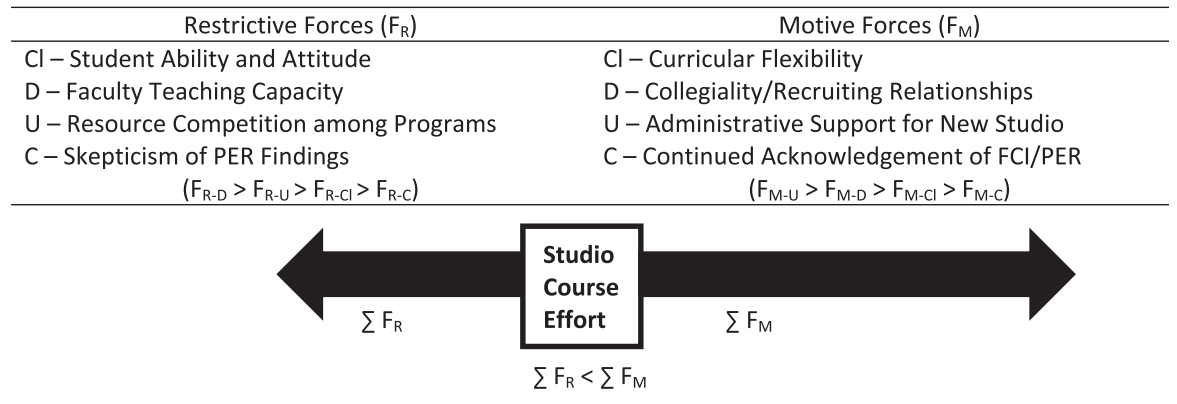

FIG. 4. Free body diagram for stage 2: Expansion of the studio physics program (Cl—classroom; D—department; U—university; C-culture). 
student-teacher instructional interactions that occur, while not specifying a particular curricular approach. With this allowance, the faculty group accessed other PER resources to engage their students in different activities that could accommodate the variation in student ability present in both courses, thus providing the group with a positive, motive force. This willingness to be flexible made it possible for these teachers to respond not only to challenges on a daily or weekly basis, but also provided the capability to make changes in new semesters to address issues that arose in previous ones. The following summary of comments came from an observation in which Robert described "flexibility" as a necessary characteristic for instructors if they were to successfully respond to the wide range of preparedness of students entering his studio physics class. This set of observations occurred during a professional development seminar that Robert was leading with several local K12 science teachers.

- Robert: Tells teachers about trying to teach this content in the physics II course, using the diagnostic exam and learning about the lack of high school physics among his students.

- Describes the low learning gains and now he is going to use PBI [Physics by Inquiry] instead because [students] are so poorly prepared.

- States he has two options-he can complain about [unprepared students] or he can adapt to the students coming into his classroom.

- Asserts that to improve the preparation of students, teachers are key and compliments the teacher for being in the class.

(External observation, July 2010)

Robert subsequently used a PBI unit concerning dc circuits in his course. Robert had originally used curricular resources that were provided by the manufacturer of some of the laboratory equipment, but the technicality of those materials presented challenges in getting students to engage. However, to maintain the level of rigor appropriate for the course (and engineering and physics majors in the class), he further supplemented his curriculum with demanding, conceptually oriented quizzes and online homework question systems developed by PER colleagues at other institutions. Albert, Isaac, and Max also used resources PER resources from colleagues at other institutions and evidence-based resources, such as Tutorials in Introductory Physics [18].

The interaction of forces acting at the classroom level during this phase of the studio course created pressures at the department and university level. As the studio courses developed waiting lists of students, the need for more sections of the courses, particularly the first course in the sequence, became apparent. However, at the university level, accommodating this demand met resistance due to tension with other programs that used the studio space.
Other programs viewed the technological capabilities of the studio classroom as ideal, even though the instructional approaches used by these departments remained largely traditional. The resistive force of space competition with these programs was overshadowed by the most pressing resistive force at this stage, the lack of new faculty to teach. The initial group (Robert, Albert, and Isaac) were limited in their ability to commit more of their time to the studio effort in light of their other responsibilities, including their research and departmental service.

The restrictive pressures the faculty group experienced during the second stage were overcome by two larger motive forces that worked in opposition. The studio faculty group was able to recruit a new member, Max, to teach another section of the studio course. The motive force that facilitated this recruitment involved the general collegiality that existed in the physics department among the entire faculty.

They want to vote yes [on new ideas for teaching, policy and outreach] unless there is a really obvious reason they have to vote no. That is the full faculty, even the people who don't agree with what we are doing. (Robert, group interview)

This atmosphere of mutual respect in the department extended to the studio effort, resulting in a general, if somewhat ambivalent, support for the studio course effort as a whole. Personal relationships encouraged by this departmental sense of respect served as the conduit for the studio faculty group to recruit a new member. Given this climate, Isaac was comfortable engaging Max in extended conversations about the effectiveness of the studio model in comparison to traditional approaches to teaching, with Isaac even having Max come to his class to "guest teach" so he could have first-hand exposure to the course. The eventual addition of Max to the studio faculty group, stemming from the departmental collegiality, was a major motive force in accelerating the studio course effort.

A second, even larger, motive force emerged around this same time for the studio course effort. The university administration noted the competition for the studio classroom space and granted a request from the studio faculty for the creation of another studio classroom. The administration provided a sizable investment of funding resources to redesign an existing instructional area into another studio classroom in the SCALE-UP model, as well as providing nonrecurring funds to purchase an entire new set of instructional laboratory equipment, including specialized pieces like oscilloscopes and low-friction cart tracks.

It may be worth mentioning that the proposal to buy equipment for the traditional class was turned down.... 
[The administration's] support is specific to what we are doing....They want to support pedagogical reforms in Physics. (Robert, group interview)

The primary motive forces outlined above (administration's investments and the departmental collegiality) were buttressed by another motive force operating at the cultural level. The acknowledgment of the studio effort's success relied on the disciplinary cultural acceptance of the validity of the Force Concept Inventory (FCI) as a measure of conceptual learning. Cultural resistance existed in the form of general skepticism to PER findings, often stemming from a reticence by some faculty to invest the necessary time and attention in light of competing research demands and specific criticism involving shortcomings of the FCI [19-22]. The studio faculty members encountered some of this PER skepticism in their discussions with colleagues, particularly with regard to topic coverage entailed in the FCI. However, the pervasiveness of the assessment's use in demonstrating differences in physics learning across multiple contexts [23] provided further support for this physics studio course program by offering validation of the measure from a broader community of physicists.

\section{Stage 3: Stabilization}

The physics studio course effort experienced two major accelerations (stages 1 and 2) and then shifted into a phase of stabilization in which the external forces shaping the program neither accelerated nor slowed the program (stage 3). During stage 3, the primary motive force supporting the physics studio revolved around the efforts of the four faculty members involved at the classroom level. (See Fig. 5.) The collaboration of this team served as a critical resource for ideas and suggestions for improving the course. Their ability to share curricular and pedagogical information helped them to maintain a stable program.

I get insights pretty much all the time. Whenever I ask Isaac something, he tells me something new pretty much. Like today, he gave me an interesting hint on how you grade homework and miniquizzes, and that you have students write things down in words. (Max, individual interview)

Indeed, the support provided by the faculty group facilitated a relatively easy transition for Max during his first semester of teaching studio. Through the help of Albert and Isaac, Max was able to achieve similar learning gains on the FCI with his students $(\sim 50 \%)$ in the studio course, continuing the trend of studio students performing better on the FCI than their peers in the traditional courses (average learning gain $\sim 20 \%$ ). The difference in learning gains across the two course formats emerged at the onset of the studio program. The time and effort invested in thinking about and working with each other to improve activities and assessments on behalf of the members of the studio faculty group provided the necessary support for the studio program to quickly establish and maintain this level of success on the FCI, which further demonstrated the effectiveness of this SCALE-UP implementation.

The curricular sharing occurring among the physics studio faculty members became especially important in light of restrictive forces operating at several contextual levels at the same time (Fig. 5). The studio physics program faced continued challenges in regards to scheduling and access to the actual studio classroom, which was exacerbated by ongoing lack of understanding by the university registrar's office. This office controlled the scheduling of courses in all classroom space on campus, yet the typical formulations they employed for making assignments did not accurately represent the needs of the studio course. The desired time block for a studio physics course entailed three weekly sessions, distributed uniformly throughout a week, meeting approximately three hours for at least two sessions.

Competing with other programs and schools for the studio space, the physics studio courses had a constantly changing schedule, with both introductory studio physics courses forced into several different scheduling scenarios. For example, during one semester, the second studio physics course was only given two and a half hours on two afternoons in the studio space and another hour of class

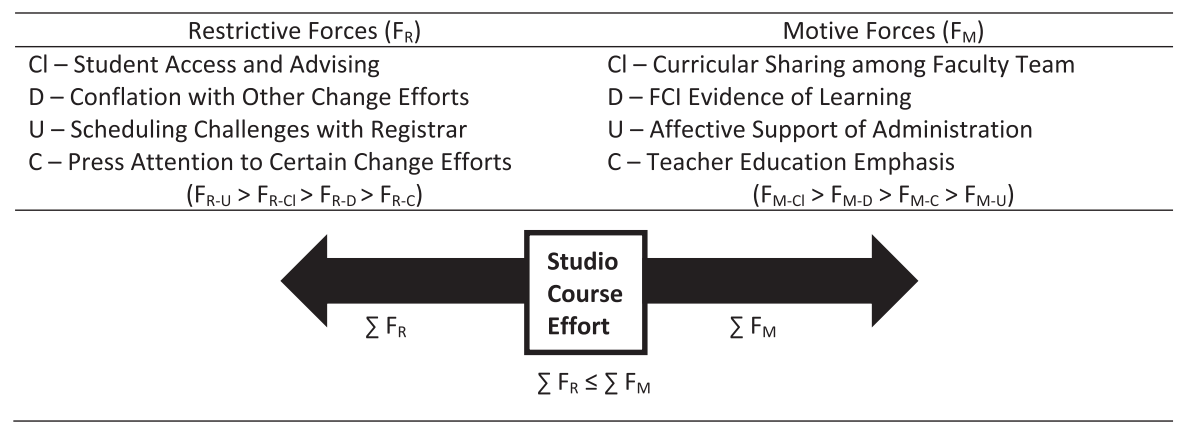

FIG. 5. Free body diagram for stage 3: Stabilization of the studio physics program (Cl—classroom; D—department; U—university; C-culture). 
time in a completely different room. Several Email conversations, appeals to administrators, and direct meetings with registrar officials did not produce any demonstrable change in this institutional relationship. The scheduling challenges fostered by the registrar's actions also created issues at the classroom level by affecting the access students had to the course, which became limited due to the odd time structure for class meetings. Further activities of other institutional programs created more resistive force that extended back down to the classroom and departmental level.

We thought we had good relations with the dean [of another college], and when we inquired about possibly working out how to keep our classes, how to offer the classes we need at the times we needed to offer them, we got essentially no response. (Albert, group interview)

We now have hard data demonstrating that the scheduling situation in [the studio space] has damaged our students' learning... There is no input channel for [the registrar] to work that into the scheduling algorithms. (Group Email conversation \#19)

The classroom level issue of student access became further compounded by a centralization of student advising resources within the college administrative offices. The repositioning of those responsibilities created communication barriers in advising students to take the studio courses. A newly established science teacher preparation program encouraged students to take the studio physics course, particularly due to the potential for those students to experience innovative, discipline-based pedagogy. However, college level advisers were not aware of these recommendations and advised students to take the traditional courses.

Furthermore, intense attention given to the science teacher preparation program created a sense of confusion among the group's departmental colleagues for the studio physics faculty members. These colleagues responded to ideas about improving and expanding the studio physics program with reservations related to the amount of attention and support already being given to the science teacher education program. The conflation of the two programs created challenges in the perception of resource needs for the studio physics program in the department.

Although resistance to the studio physics program developed at the department level, the studio physics faculty group was able to respond with a motive force emerging from the several semesters of studio courses that had occurred. Since its beginning, the studio physics group continuously administered the FCI at the beginning and end of each semester in the first introductory course. The FCI was similarly administered in the traditional lecture course. The normalized learning gains achieved on the FCI were far greater for the studio physics program than those from the lecture format, even considering that Albert taught a traditional lecture section after having taught the studio version. This pattern held even when Albert was an instructor in the traditional classroom. These FCI results became critical evidence for the studio physics faculty group to demonstrate the effectiveness of the studio program to departmental colleagues and administrators. The studio faculty group privileged the FCI evidence for learning and often used this information to describe the studio program to colleagues and the achievements that are possible in studio courses.

We have proof in the FCI, and therefore, people who...still teach in a lecture setting...the fact is that that method results in students having lower learning outcomes. (Albert, group interview)

The FCI evidence provided enough indication of success for continued administrative approval at the university level. This motive force, though somewhat perceptually muted during this stage, was enhanced by a cultural motive force concerning growing calls for improving the teacher preparation mission of large research universities. These calls emphasized the need for future science teachers to experience innovative pedagogy in their education and disciplinary content courses. The studio physics program offered university administrators an example of how this institution was meeting those calls. However, at the same time, another cultural force emerged, acting in resistance to the studio course effort, which concerned greater press attention to another instructional innovation on campus that focused on infusion of technology in lecture classrooms. The resistive nature of this attention was due in part to the limited impact of such changes when compared to more transformative efforts like the studio physics program. The cultural attention given to these technology efforts took public attention away from the studio effort, and this publicity provided impetus for increased administrative attention and support [5].

\section{Stage 4: Diffusion}

The first three stages of motion in the studio course effort described above occurred during the data collection and analysis phase of this study. The fourth stage, diffusion (shown in Fig. 6), represents a prediction of the resistive and motive forces expected to continue to influence the trajectory of the studio physics program. Although these are predictions of future events, they are grounded in observations and data collected during the previous stages.

At the classroom level, the issues of student access and their academic ability will continue to shape the development of the studio physics program. Yet, the motive and resistive dimensions of these factors can be difficult to predict in a reasonable manner. Another broad force that was observed to play a critical role at the classroom level in previous stages involved the curricular and assessment 


\begin{tabular}{|c|c|}
\hline Restrictive Forces $\left(F_{R}\right)$ & Motive Forces $\left(F_{M}\right)$ \\
\hline $\mathrm{Cl}$ - Limited Array of Learning Measures & $\mathrm{Cl}$ - Flexibility to Explore Enhancements \\
\hline D - Faculty Reticence to Time Investment & D - Support among Studio Faculty \\
\hline U - Availability of Studio Space & U - Plans for More Studio Spaces \\
\hline C-Limitation of PER Resources & C - Focus on STEM Education \\
\hline \multirow[t]{3}{*}{$\left(F_{R-U}>F_{R-D}>F_{R-C}>F_{R-C l}\right)$} & $\left(F_{M-D}>F_{M-C I}>F_{M-C}>F_{M-U}\right)$ \\
\hline & $\begin{array}{l}\text { Studio } \\
\text { Course } \\
\text { Effort }\end{array}$ \\
\hline & $F_{R}<\sum F_{M}$ \\
\hline
\end{tabular}

FIG. 6. Free body diagram for predicted forces in stage 4: Diffusion of the studio physics program (Cl—classroom; D—department; U-university; C-culture).

resources available to the studio physics faculty group. The FCI scores from several semesters provided evidence of meeting the group's learning goals, at least in the first course, as demonstrated by its frequent use in conversations about the studio physics program's success. On the other hand, the need for well-accepted and validated assessment instruments addressing topics not on the FCI (especially for electricity and magnetism) will become a restrictive force for the studio program during stage 4 . In working to solve this challenge, the faculty group had begun experimenting with the use of other assessments, such as the BEMA [24] and DIRECT [25] assessments during stages 2 and 3 , and in stage 4 , to determine the most appropriate approach to move the studio physics program forward by providing a more comprehensive body of evidence for achieving the learning goals.

At the departmental level, the studio physics faculty group faces its greatest resistance in the limited number of colleagues interested in teaching a studio course. In light of the research and publishing demands ubiquitous on research university campuses, many department faculty members viewed the perceived time investment involved in teaching a studio course as prohibitive. The studio faculty group was entirely composed of scholars who had achieved tenure. To counteract the time demands for studio, the studio physics faculty group was able to use their collaborative relationship for support. As Max joined this group, Albert and Isaac, especially, were able to provide him with curricular resources and advice on course logistics, preventing him from spending excessive time preparing for the course on his own. The supportive nature of this group, acting as a motive force in the department, also serves as a recruitment technique in which the studio faculty members would engage potential new members, offering them opportunities to guest teach in their courses. These efforts had begun to show progress, such as with Isaac getting increased positive feedback from another colleague he was actively recruiting.

The biggest potential barrier I see is just to try it [teaching a studio course]. You have to overcome this natural kind of feeling "Well, it's something new. I'm not sure if it will work for me." It will. You just have to take some effort just to start. (Max, group interview)

University level forces operating on the studio physics program at this stage also include the competition for resources from other programs on campus, and administrative issues from the registrar. However, perhaps the most fundamental university level force affecting the future diffusion of the studio physics program involves the actual existence of studio classroom space that meets the design elements of the SCALE-UP model. At the end of this study, the two existing studio classrooms were already used to their scheduling limits, creating a resistance to an expansion of the program. Yet, observation and interview data were collected that described the acceptance and promotion of building plans for a new science classroom building that included several more studio classrooms. This plan was discussed by a committee of university science faculty, including Robert and Isaac, and the inclusion of studio spaces was supported by a number of colleagues. The realization of this new building exists as an essential motive force for future expansion of the studio physics program. The following excerpt was observed during a meeting of a university committee tasked with the development of plans for a new academic laboratory and science teaching space. Robert and Isaac were not the only members of the university community who argued in support of including multiple studio classrooms in this newly proposed building. The following observation note provides a summary of events that took place during the discussion.

- Faculty \#1 argues for lecture halls and studio rooms for phase 1.

- Faculty \#2 present asks what a SCALE-UP room isRobert and Isaac describe the room design.

- Faculty \#1 argues again to the starting idea with lecture hall and SCALE-UP room for the phase 1 building.

- [Researcher Note: No other committee member is raising an issue with the inclusion of several studio rooms in the phase 1 plan (up to five rooms).] (External committee meeting observation, December 2010) 
Culturally, the external forces acting upon the studio physics program relate more to broad issues arising in the national conversation about innovation in higher education. National organizations of many allegiances continue to emphasize the need for increased production of STEM majors and graduates. This increased attention, and the potential for associated funding resources, offers a fertile context for the studio physics program to position itself as a meaningful response to those calls. The studio faculty group, especially Robert, had already incorporated several of these reports into the justification for the studio physics program. In contrast, a limiting cultural force developed from the lack of acknowledgement of the achievements of PER innovations $[9,26]$, not only within the physics community, but in broader education circles. An intense use of only PER resources by the studio physics program could continue to isolate the effort from those broader discussions. That same intense focus could also limit the pool of resources the studio faculty group draws from as they continue to improve the studio physics program.

\section{DISCUSSION}

The studio physics faculty group endeavored to reshape the way introductory physics courses at their institution were taught in order to improve student engagement and learning, using evidence-based models and ideas from the PER community, specifically the SCALE-UP model [12]. Over several years, the studio physics program has developed from a single section of the first course to a steady offering of several sections of both courses with potential for further expansion. However, this effort did not exist unto itself. The development and implementation of the studio physics program ensued within a complex collection of institutional structures and cultures. Contextual factors shaping the scope and nature of the studio physics courses arose in multifaceted ways reflecting the interconnected environment.
Researchers from the PER community and other organizations have called for further research into the contextual factors that influence the adoption of evidence-based, PER innovations $[1,6,11]$. The implementation of PER innovations is influenced heavily by individual factors stemming from the instructors who teach the courses being changed [9]. Yet, research has also demonstrated that these individual factors do not fully account for the lack of adoption on their own. Theoretical models emerging from this work emphasize that both individual and situational factors need to be transitioned from more traditional states to being more alternative [6].

The group of faculty members who enacted this change in their introductory course sequence came together out of a shared commitment to more engaging instruction that produced deeper conceptual understanding of physics in their students. This group's structure and interactions supported the shifting of individual factors indicated as critical elements of theoretical change models $[6,8]$. The study described here identified situational factors that both benefited and limited the development of a PER-based studio physics program within a large, research university.

The results of this study support findings of other analyses that emphasize the importance of time and sustained engagement with change efforts [9]. The studio physics program grew over several years, beginning with simple ideas and desires on the part of concerned faculty members. During that time, the studio physics program experienced developmental pressures from several sources, and the nature of those forces also changed in magnitude and kind over that time. The interactions among programs on campus at times provided useful support for the creation of a studio classroom, yet at other times fostered tensions as they competed for similar resources, including the studio room itself. Temporally, administrative approval of the studio physics program provided a minimum level of continuous support, yet specific instantiations, particularly in the form of funding infusions, were far more crucial.

Beyond the overarching situational factor of time invested in a change effort, the results of this study also

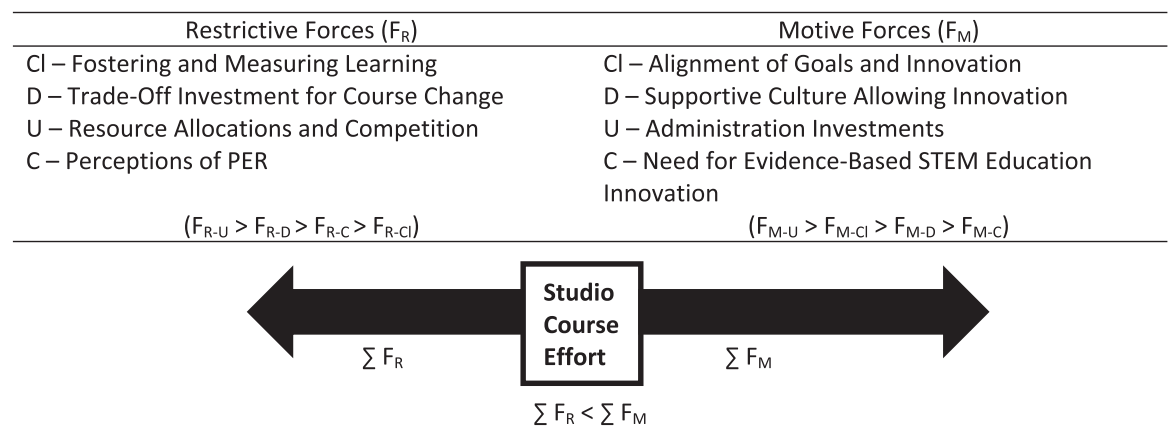

FIG. 7. Free body diagram summarizing the external forces influencing the studio physics program (Cl—classroom; D—department; $\mathrm{U}$-university; $\mathrm{C}$-culture). 
provide evidence for broad forces that operate at several different contextual levels across different stages of time, some motive and some resistive in direction. Figure 7 provides a summary free body diagram highlighting both kinds of forces acting on the studio physics program for contextual levels aligned with the TCSR framework [8] used for analyzing the data for this study. These broader forces generally work in opposition to each other directly. However, the influence of these forces can reach across organizational boundaries to affect events occurring in other interconnected levels. The recognition of these more fundamental factors further encourages consideration of implications for change efforts at similar institutions, which will be discussed within each section below.

\section{A. Classroom level factors}

The studio physics program was fundamentally generated from contextual forces operating at the classroom level. A lack of meaningful physics learning in their students, observed by all four members of the studio physics faculty group, led each of them to seriously reflect on their teaching and the kind of learning environment they provided. This sense of discontentment with pedagogy and the willingness to consider alternative options has been indicated elsewhere as a necessary initial component to any successful change effort $[8,9,17]$. These faculty members desired a learning environment where the students were more engaged in investigative learning and conceptual discussions with each other and the instructors.

The coalescing of this group in support of a studio physics course demonstrates an aspect of the primary motive force operating at the classroom level in this study. The alignment between the studio physics faculty group's desired learning goals and the PER innovations facilitated a deep commitment to the success of the program. The SCALE-UP model which guided the studio physics program entailed a complete physical redesign of the classroom space. This type of physical environment was more suited to the types of learning interactions the faculty members wanted to have with their students. Thus, at the classroom level, the alignment of these two fundamental elements offered a solid foundation from which the studio course effort could build. Although this agreement occurred in a broader scope, a lack of similar alignment in more specific instances demonstrates the resistive aspects of this contextual influence.

As the studio physics program developed, the four faculty members came to privilege and emphasize the FCI assessment data as evidence for the success of the program in terms of increased student learning. However, the faculty group understood that the FCI concerns only a portion of physics content material addressed in the first introductory course. In hopes of building further evidence of success, the faculty group continued to struggle with finding accurate ways of measuring the learning of other physics concepts. Robert attempted using several different assessments to measure his students' learning of electricity and magnetism concepts. Similarly, all of the instructors were limited in explicitly defining their learning goals and targets for their students, complicating the ability to measure learning achievement beyond basic metrics of test and quiz scores. This issue further impacted the ability of the studio faculty group to understand the effectiveness of other evidenced-based PER curricula they incorporated into their studio physics program.

The tension arising from these different aspects of alignment between learning goals and PER innovations relates to other classroom level factors identified in the literature [6]. Apprehension regarding implementing PER innovations and the feeling that physics content material addressed is overall lessened can be managed if the learning goals for a course are explicitly described. Faculty who choose to implement PER innovations should be clear about exactly what they want their students to learn and how the innovation will help them achieve those goals. That said, this creation of defined learning goals can also provide an opportunity for the faculty to discern the kind of understanding they desire (i.e., memorization of a broad collection of physics information within topical categories or ability to apply more fundamental, guiding physics concepts). Committing to learning goals of a particular character could also alleviate stress regarding the volume of material addressed in a course where PER innovations are implemented. Making these learning goals clear could possibly assist in improving students' attitude towards the change efforts, another important classroom level factor found in this study and others [6].

\section{B. Department level factors}

The contextual level influences involving the Department of Physics at the institution described by this study invoke considerations of course sequences selected by department committees or formalized degree programs supported by the faculty members. Yet, the most influential forces affecting the studio physics program in the department emerge from the respectful culture that exists among the diverse group of scholars. In a general sense, the supportive culture that existed among these colleagues allowed for the collaborative group of Robert, Isaac, and Albert to come together and work earnestly on developing the studio physics program. The program was implemented and expanded under the auspices of collegiality that existed within the department.

The respect shared among the faculty in this department gave rise to the primary resistive force through the creation of unstated boundaries. As the studio physics program grew, the original three faculty members were taxed as they worked on its enhancement while still maintaining their other departmental responsibilities. In searching for others to join their effort, several colleagues expressed 
tepid interest, yet they also were reticent due to the perceived investment of time and effort to change their way of teaching. The respect of the department was not garnered through an individual member's teaching ability; rather, the research agenda executed by a colleague was often treated as the primary measure of value, as is common in many large, research universities [15]. Indeed, the studio physics faculty group was composed completely of tenured faculty with established research agendas, a distinction that the group itself agreed was necessary for the investment of focus and time needed to switch to teaching studio.

Although this investment of time and focus served as a resistive force at the department level, the collegial culture among the faculty members in the department also fostered the motive, supporting force that helped the studio program expand. As a whole, the department membership would support any colleague's ideas if they were well thought out and the associate was earnest in their interest. Although several colleagues had expressed reservations about the effectiveness and utility of the studio program at presentations concerning it, they were still supportive of the studio faculty group if that was how they chose to invest their time. Again, it should be emphasized that the departmental culture that existed gave rise to the core studio physics faculty group. The personal relationships that existed within this group became critical resources in supporting the studio course effort and challenges it faced. The newest member of the group, Max, was recruited to join the studio physics program through the collegial friendship he had developed with Isaac. The challenge of Max's initial investment of time and focus in switching to the studio course was alleviated through the support of the other group members, providing him with curricular materials and logistical advice. Thus, the departmental culture of mutual respect and support facilitated this kind of team effort, even if the entire faculty did not share the group's approach to physics teaching.

The departmental culture is shaped, in part, by department administrators. The chair of a department can greatly influence the work that is valued in the department, through policy actions and public support [3]. The studio physics faculty group experienced a continued level of support for their efforts across two separate department chairs. Admittedly, Albert was serving in the role of department chair when the studio program was being initially developed, which provided critical financial and administrative assistance. However, the next colleague to be elected to the chairmanship continued this support with strong affirmations towards the program and the studio faculty group, and continuing to encourage the expansion of the studio physics courses. An administrator can shape other factors that have been identified as potential barriers to implementation of innovations, including the time structure allotted to different courses and positive recognition of these kinds of efforts $[6,8]$. This study provides further support for the need for department administrators to assume these leadership activities, and institutional professional development should be provided to enhance chairs' understanding of their leadership responsibilities [3]. The further education for leadership can serve chairs and deans in creating an institutional culture that supports implementation of innovations.

\section{University level factors}

Administrative leadership resonates across contextual levels as an underlying element for promoting meaningful educational change $[3,27]$. University administrators, including deans and provosts, can provide leadership in creating an innovative academic culture by publicly supporting and highlighting successful change efforts, implicating their success in the overall institutional mission. These administrators also lead through the provision of resources under their responsibilities in facilitating the development and expansion of these efforts.

The major motive force supporting the studio physics program encompasses the collection of critical investments made into the effort on the part of various university level administrators. The specific nature of PER innovation, such as a redesigned studio classroom space, determines the type of administrative support needed for success. The studio physics effort benefited from the financial advantages it offered in requiring minimal shifting of faculty teaching and teaching assistant resources. The model developed by the studio physics faculty group also offered a slightly more amenable cost-to-benefit ratio when comparing the faculty hours used to the student credit hours generated for the studio physics program versus the traditional lecture-laboratory-recitation course combination. Thus, the major financial investments provided by university administrators involved nonrecurring expenses that have less long-term budgetary implications. Another benefit of those investments for the administrators involved the appeal of these investments to other programs on campus, providing further justification for the necessary expenditures.

Although the intracampus appeal of the studio spaces helped garner university administrative support, that appeal also engendered competition for institutional resources that acted as a resistive force for the studio physics program. The use of the studio classroom by other academic programs persisted throughout the entire study as a source of difficulty in securing appropriate scheduling blocks for the studio physics courses from the university registrar. The studio faculty group brought this issue to the attention of several administrators, including deans and vice presidents, in attempts to develop workable solutions, but they received little in the way of meaningful responses. A coordinating committee composed of members from all interested programs was originally proposed to help determine how the studio space resource should be allocated. 
However, once the studio space was established, this guiding committee was never formalized and given authority over the space. The emergence of these opposing forces acting on the studio physics program offer insight into another set of critical considerations for implementing educational innovations in large university contexts.

University administrators and their acts of leadership play a key role in determining the success of implementing meaningful educational changes. As a concrete show of support, administrators can steer necessary financial and logistical support towards innovative efforts that have an evidence base for improving teaching and learning. Concrete policy decisions involving class spaces, scheduling, and obtaining necessary curricular resources for change efforts have been identified as factors that administrators can directly remove as barriers to change [3,6]. These actions are critical and should be concurrent with further leadership actions publicly promoting programs like the studio physics courses as vital to advancing institutional goals. The more abstract leadership roles that university administrators can assume involve promoting and giving special recognition to improvement efforts $[6,11]$.
Tensions caused by competition between programs on campus for limited institutional resources demands administrative leadership in taking an active role in alleviating those tensions and working towards consensus solutions. University administrators can focus their efforts on working towards system compatibility between existing institutional structures and those accessed by change efforts [9]. Again, university administrators should engage in professional development to enhance their capacities in these areas [3].

\section{Cultural level factors}

Change efforts are shaped by the cultural contexts in when they occur, often represented by broad societal and political conversations focused on the nature of postsecondary education. These conversations, although removed, become essential as change agents seek justification and validation for their work [3]. The escalating national conversation about improving STEM education, involving a variety of stakeholders, created a background dialogue that the studio physics group was able to draw upon to justify their local effort at improving introductory physics

TABLE II. Contextual factors affecting implementation and maintenance of a change effort. Recommendations are based upon evidence drawn from the study, but may not reflect the entirety of approaches available for specific educational contexts.

\begin{tabular}{|c|c|c|}
\hline Contextual level & Influential factors & Considerations for change efforts \\
\hline Classroom & $\begin{array}{l}\text { Alignment of change } \\
\text { ideas and efforts }\end{array}$ & $\begin{array}{l}\text { - Create explicit learning goals or desired outcomes that will guide the } \\
\text { evaluation of the change effort } \\
\text { - Consider the change or innovation to be implemented and make sure it } \\
\text { is designed to meet the determined goals or outcomes } \\
\text { - Determine the most appropriate measures for evaluating the success of } \\
\text { achieving those goals or outcomes }\end{array}$ \\
\hline Department & $\begin{array}{l}\text { Faculty collegiality within } \\
\text { the department }\end{array}$ & $\begin{array}{l}\text { - Establish a committed team focused on implementing change that can } \\
\text { support each other in the endeavor } \\
\text { - Prepare evidence and arguments to help skeptical colleagues under- } \\
\text { stand the goal of the change and appreciate the sincerity of the effort } \\
\text { - Work with the chairperson to develop public and financial support for } \\
\text { the change effort } \\
\text { - Be mindful of the financial requirements and budgetary implications } \\
\text { of the change effort }\end{array}$ \\
\hline University & $\begin{array}{l}\text { Allotment and management } \\
\text { of finite resources }\end{array}$ & $\begin{array}{l}\text { - Develop partnerships and support with institutional administrators to } \\
\text { obtain needed resources for the change effort } \\
\text { - Prepare arguments highlighting how the change effort serves broader } \\
\text { institutional goals and mission } \\
\text { - Engage administrators to provide management and guidance in work- } \\
\text { ing with other institutional entities and sharing resources }\end{array}$ \\
\hline Cultural & Broad societal perspectives & $\begin{array}{l}\text { - Be aware of national discourse salient to the goals and work of the } \\
\text { change effort } \\
\text { - Use the statements and findings of national organizations to bolster } \\
\text { supportive arguments for the change effort and temper the restrictive } \\
\text { arguments } \\
\text { - Promote the change effort and seek advice among professional networks }\end{array}$ \\
\hline
\end{tabular}


courses. Robert, particularly, was skilled in invoking recommendations from national level reports about the shortage of physics teachers and the need for more effective physics education to argue for the expansion of the studio physics program.

However, in a similar manner, earnest critiques and reservations about aspects of some innovations stemming from scholarly works of other physicists, such as the limitations of the FCI, also enhanced the resistive forces on the studio physics program. That is not to say that these reservations and critiques should be eliminated. Rather, they rightfully temper these broad conversations and aid in refining innovations, but such critiques can be used as a source for creating barriers. Those barriers can engender thoughtful criticism, but they should not be purposed for broad based condemnation of educational innovation.

Professional organizations of scholars and researchers offer opportunities for recognition of innovative programs, and should strive to continue to do so $[3,6]$. These efforts should continue to expand as they can begin to shift the perception of what is privileged within professional cultures. The general acceptance of the FCI as a valid measure of learning demonstrates the impact of the PER community [11]. The continued use and privileging of this instrument by PER researchers provided the studio physics faculty group with evidence to argue for the effectiveness of the studio physics course that was compelling for their institutional colleagues. This study offers a case example of how, although seemingly removed at the level of more local considerations, broad societal conversations become fundamental anchors for justifying and working on educational innovations.

\section{E. Summary}

The findings of this study reflect interactions spanning multiple institutional contextual levels and an extended period of time. The influential forces described in Fig. 7 above represent tensions stemming from operational realities of large, research universities. Foremost, time allowance for any change effort, and the provision for it, will always serve as a fundamental factor that shapes that development of projects. Beyond time, change efforts in large, research universities will also encounter other tensions at multiple levels within their contexts. Table II provides a list of the major tensions at each of the levels analyzed in this study. The recommendations listed are inspired by activities observed during the course of this study. However, as discussed elsewhere in this article, every university presents a unique context with distinct collections of personalities and relationships. Thus, these recommendations may not be appropriate for every change effort.

\section{LIMITATIONS}

The study presented here provides examples of the challenges and contributions that develop during the initiation, implementation, and expansion of a studio physics course program in a large, research university context. This educational change effort stemmed from evidence-based innovations found in PER literature $[1,12]$ that demonstrated success in improving undergraduate students' conceptual understanding of physics concepts. Although the learning successes attributed to the SCALE-UP model and similar studio physics course formats had been demonstrated, researchers emphasized the need for further understanding of how these types of physics courses are successfully implemented within the complex institutional structures and cultures present at most universities [1,9,11]. This study focused on the contextual influences acting from various levels that both assisted and challenged the development of a studio physics course program. The findings discussed here should not be considered generalizable, but do provide evidence supporting general themes described elsewhere $[3,11]$. The results also offer issues for similar institutions to consider as faculty members embark on implementing their own innovations.

Implications resonating with the forces acting at the classroom, department, university, and cultural levels have been described above. In regard to the study overall, the findings discussed are specific to the implementation of a studio physics course model within a large research university context. The particular nature of this PER-based innovation invokes the coordination of efforts at multiple contextual levels within an institution, creating a unique classroom space and a commitment by faculty members to using meaningful alternative physics pedagogy. Not all endeavors in educational change will necessitate such direct interactions with other department and university entities, thus the types and nature of contextual influences will differ.

The results of this study are also limited by nature of the research subjects. The TCSR theoretical framework employed in analyzing the data is admittedly grounded in the perceptions and actions of the teacher. Although the studio physics program comprises a multifaceted effort within a complex system, the thoughts and activities of the faculty members involved in its creation exist as the primary drivers of the entire program. Thus, their perceptions of the influences acting upon their program are fundamental to its actual development. The data collected were drawn almost exclusively from the studio physics faculty group, but the results of this study would be enhanced by further collection of data relating first-hand accounts and impressions from actors tied to the contextual influences from other levels, such as chairs and deans. Research that continues to explore these issues should also work to incorporate the multiple perspectives that can be accessed, and frameworks that can accommodate multiple perspectives would be useful developments for further studies. 


\section{ACKNOWLEDGMENTS}

The authors offer sincere gratitude to Albert, Isaac, Max, and Robert for their willingness to share their thoughts, beliefs, and experiences with regard to the studio physics course program. The authors would also like to thank Victor Sampson, Michael Dentzau, and Ellen Granger for their thoughtful critiques of earlier versions of this manuscript and input for revisions.
[1] J.L. Docktor and J. P. Mestre, A synthesis of disciplinebased education research in physics, in Proceedings of the Second Committee Meeting on the Status, Contributions, and Future Directions of Discipline-Based Education Research, 2011, http://www7.nationalacademies.org/ bose/DBER_Docktor_October_Paper.pdf.

[2] M. H. Dancy and C. Henderson, Pedagogical practices and instructional change of physics faculty, Am. J. Phys. 78, 1056 (2010).

[3] A. Austin, Promoting evidence-based change in undergraduate science education, in Proceedings of the Fourth Committee Meeting on the Status, Contributions, and Future Directions of Discipline-Based Education Research, 2011, http://www7.nationalacademies.org/ bose/1DBER_Austin_March_Paper.pdf.

[4] C. Henderson and M.H. Dancy, Physics faculty and educational researchers: Divergent expectations as barriers to the diffusion of innovations, Am. J. Phys. 76, 79 (2008).

[5] D. Sunal, E. Wright, and J. Bland-Day, Research in Science Education: Reform in Undergraduate Science Teaching for the 21st Century (Information Age Publishing, Greenwich, CT, 2004).

[6] C. Henderson and M.H. Dancy, Barriers to the use of research-based instructional strategies: The influence of both individual and situational characteristics, Phys. Rev. ST Phys. Educ. Res. 3, 020102 (2007).

[7] M. Eley, Teachers' conceptions of teaching, and the making of specific decisions in planning to teach, Higher Educ. 51, 191 (2006).

[8] J. Gess-Newsome, S. Southerland, A. Johnston, and S. Woodbury, Educational reform, personal practice theories, and dissatisfaction: The anatomy of change in college science teaching, Am. Educ. Res. J. 40, 731 (2003).

[9] C. Henderson, A. Beach, and N. Finkelstein, Facilitating Change in Undergraduate STEM Instructional Practices: An Analytic Review of the Literature, J. Res. Sci. Teach. 48, 952 (2011).

[10] M. Devlin, Challenging accepted wisdom about the place of conceptions of teaching in university teaching improvement, Int. J. Teach. Learn. Higher Educ. 18, 112 (2006), http://www.isetl.org/ijtlhe/pdf/IJTLHE89 .pdf.

[11] National Research Council, Promising Practices in Undergraduate Science, Technology, Engineering, and Mathematics Education: Summary of Two Workshops (The National Academies Press, Washington, DC, 2011).
[12] R. Beichner, J. M. Saul, D. S. Abbott, J. J. Morse, D. L. Deardorff, R. J. Allain, S. W. Bonham, M. H. Dancy, and J.S. Risley, The Student-Centered Activities for Large Enrollment Undergraduate Programs (SCALEUP) project, in Research-Based Reform of University Physics, edited by E. F. Redish and P. J. Cooney (American Association of Physics Teachers, College Park, MD, 2007), Reviews in PER Vol. 1.

[13] P. Laws, Workshop Physics (2002), http:// physics.dickinson.edu/ wp_web/WP_homepage.html.

[14] K. Cummings, The Rensselaer Studio Model for Learning and Teaching: What have we learned? in Proceedings of the National Research Council's Workshop Linking Evidence to Promising Practices in STEM Undergraduate Education, 2008, http://www7.nationalacademies.org/ bose/Cummings_CommissionedPaper.pdf.

[15] R. Geiger, Knowledge \& Money (Stanford University Press, Stanford, CA, 2004).

[16] A. Strauss and J. Corbin, Basics of Qualitative Research: Techniques and Procedures for Developing Grounded Theory (Sage Publications, Thousand Oaks, CA, 1998), 2nd ed.

[17] S. Southerland, S. Sowell, and P. Enderle, Science teachers' pedagogical discontentment: Its sources and potential for change, J. Sci. Teach. Educ. 22, 437 (2011).

[18] L.C. McDermott and P.S. Shaffer, Tutorials in Introductory Physics (Prentice Hall, New York, NY, 2001).

[19] J. Docktor and K. Heller, Gender differences in both force concept inventory and introductory physics performance, AIP Conf. Proc. 1064, 15 (2008).

[20] D. Hestenes, M. Wells, and G. Swackhamer, Force concept inventory, Phys. Teach. 30, 141 (1992).

[21] D. Huffman and P. Heller, What does the force concept inventory actually measure?, Phys. Teach. 33, 138 (1995).

[22] S. Popp, D. E. Meltzer, and C. Megowan-Romanowicz, Is the Force Concept Inventory biased? Investigating differential item functioning on a test of conceptual learning in physics, in Proceedings of the annual meeting for the American Education Research Association, New Orleans, 2011 (American Education Research Association, Washington, DC, 2011).

[23] A. Savinainen and P. Scott, The Force Concept Inventory: A tool for monitoring student learning, Phys. Educ. 37, 45 (2002).

[24] L. Ding, R. Chabay, B. Sherwood, and R. Beichner, Evaluating an electricity and magnetism assessment tool: Brief electricity and magnetism assessment, 
Phys. Rev. ST Phys. Educ. Res. 2, 010105-1-7 (2006).

[25] P. Engelhardt and R. Beichner, Students' understanding of direct current resistive electrical circuits, Am. J. Phys. 72 , 98 (2004).
[26] R. Hake, Lessons from the physics education reform effort, Ecol. Soc. 5, 28 (2002), http://www.consecol.org/ vol5/iss2/art28/.

[27] M. Fullan, The NEW Meaning of Educational Change (Teachers College Press, New York, 2007), 4th ed. 